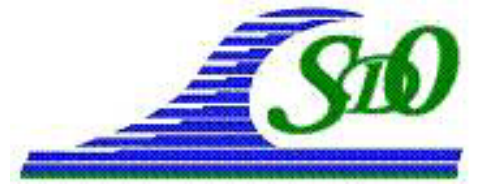

XI ìmes Journées Nationales Génie Côtier - Génie Civil

Les Sables d'Olonne, 22-25 juin 2010

DOI:10.5150/jngcgc.2010.024-C C Editions Paralia CFL

disponible en ligne - http://www.paralia.fr - available online

\title{
Trajectoires de particules et tri sédimentaire sous l'action d'un écoulement oscillant au dessus d'un fond ridé
}

\author{
Tien Dat CHU ${ }^{\mathbf{1}}$, Armelle JARNO-DRUAUX ${ }^{\mathbf{1}}$, \\ Alexander EZERSKY ${ }^{2}$, François MARIN ${ }^{1}$
}

1. Université du Havre, UFR Sciences et Techniques, FRE 3102 CNRS - LOMC, 53 rue de Prony, 76058 Le Havre Cedex, France.

tien-dat.chu@univ-lehavre.fr ; armelle.jarno-druaux@univ-lehavre.fr ;

francois.marin@univ-lehavre.fr

2. Université de Caen, Faculté des Sciences, UMR 6143 CNRS - M2C,

2-4 rue des tilleuls, 14000 Caen Cedex, France

alexander.ezersky@univcaen.fr

\section{Résumé :}

Les travaux présentés concernent une étude sur les trajectoires de particules en suspension sous l'action d'une houle stationnaire au dessus d'un fond ridé fixe. Expérimentalement, un tri sédimentaire est observé. Il se caractérise par des zones de dépôt différentes pour les petites et les grosses particules. Lorsque l'écoulement s'amortit, les petites particules se concentrent dans des zones étroites localisées à la crête des rides. Par contre, les grosses particules se déposent partout. Un modèle théorique simple est développé dans le but d'expliquer le mouvement des petites particules dans la phase d'amortissement de l'écoulement oscillant. Nous obtenons un bon accord entre les résultats issus du modèle théorique et les résultats expérimentaux.

\section{Mots-clés :}

Trajectoire de particule - Tri sédimentaire - Ride - Houle - Canal à houle

\begin{abstract}
:
This work deals with a study of the suspended particles trajectories under standing waves above a fixed rippled bed. Sediment segregation is experimentally observed. It is characterized by different deposits zones for small and coarse particles. When the flow is damping, the small particles concentrate in localized narrow zones on the ripple crests. On the other hand, the coarse particles settle everywhere. A simple theoretical model is developed in order to explain the motion of small particles when the oscillatory flow is damping. We obtain a good agreement between results issued from the theoretical model and the experimental results.
\end{abstract}

Keywords:

Particle trajectory - Sediment segregation - Ripple - Waves - Wave flume 


\section{Introduction}

En zone côtière, les fonds marins sont souvent couverts par des petites structures appelées rides (longueur d'onde typique $\mathrm{L}_{\mathrm{r}} \approx 10 \mathrm{~cm}$ ). Celles-ci sont le résultat d'une interaction très complexe entre la houle, le courant et le fond granulaire. Beaucoup d'études théoriques et expérimentales ont été réalisées (NIELSEN, 1981 ; FARACI \& FOTI, 2002) afin de comprendre les processus pilotant la formation et la morphologie des rides, mais la plupart considèrent que le fond granulaire ne contient que des sédiments homogènes en taille et en densité. Cependant, les études in-situ montrent que la taille et la densité des sédiments naturels sont très variées. Un tri sédimentaire se produit mais les mécanismes de celui-ci sont mal connus. Récemment, le développement de la technique de mesure PIV (Particle Image Velocimetry) (BALASUBRAMANIAN et al., 2008) a permis d'étudier complètement l'écoulement au dessus d'un fond ridé. Mais à notre connaissance, il n'y a pas d'étude expérimentale concernant la trajectoire des particules sous l'action d'un écoulement oscillant. Des tests sont effectués en canal à houle afin d'analyser les processus de tri sédimentaire à partir de mesures des trajectoires de sédiments en suspension au-dessus d'un fond ridé. L'influence de la taille des sédiments sur leurs trajectoires et sur leurs zones de dépôt est considérée. Les résultats expérimentaux sont comparés avec les résultats issus d'un modèle théorique que nous avons développé.

\section{Dispositif expérimental - Conditions d'essai}

Les expériences ont été menées dans un canal à houle de 5,4 $\mathrm{m}$ de longueur et $0,3 \mathrm{~m}$ de largeur, au Laboratoire Ondes et Milieux Complexes à l'Université du Havre.

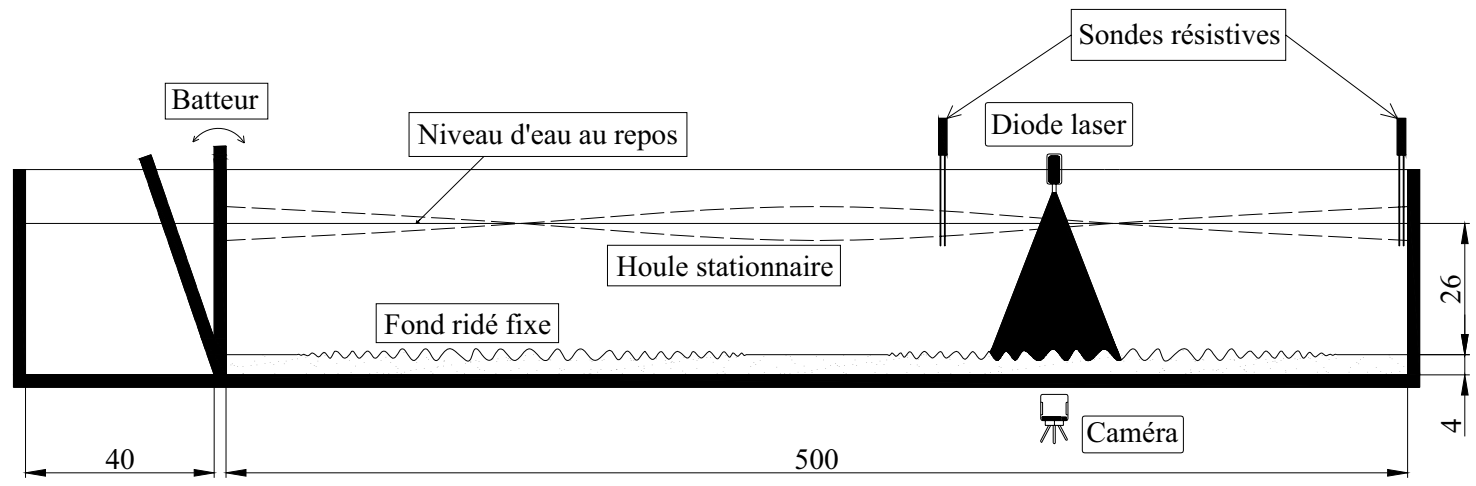

Figure 1. Canal à houle et dispositifs expérimentaux (Dimensions en centimètres).

La houle est générée par un batteur situé à une extrémité du canal utilisé en mode résonant. La fréquence du batteur est choisie très proche de la première fréquence résonante $\mathrm{f}_{1}=0,31 \mathrm{~Hz}$. La profondeur d'eau est 0,26 m. L'évolution temporelle de la surface libre est mesurée à l'aide de deux sondes résistives. La hauteur de la houle stationnaire mesure $0,04 \mathrm{~m}$. Les rides sont générées sous l'action de la houle 


\section{XI $I^{\text {èes }}$ Journées Nationales Génie Côtier - Génie Civil}

Les Sables d'Olonne, 22-25 juin 2010

stationnaire à partir d'un fond initialement plat composé de poudre de Polychlorure de Vinyle ( $\mathrm{PVC}$ densité relative $\mathrm{s}=1,35$; diamètre médian $d_{50}=0,2 \mathrm{~mm}$ ). La morphologie de rides change le long du canal. Lorsque le fond atteint son état d'équilibre, on le fixe.

Les particules sont tamisées pour les trier en gammes de taille $(100-125 \mu \mathrm{m}$; 125 $250 \mu \mathrm{m} ; 250-315 \mu \mathrm{m}$ et $400-500 \mu \mathrm{m})$. Les particules introduites sont éclairées par un plan laser vertical créé par une diode laser à He-Ne. La caméra utilisée est une caméra rapide DALSA PT 41-4M60. Sa résolution maximale est de 2352 pixels $\times 1728$ pixels, pour 62 images par seconde.

On étudie une zone proche du nœud vers-l'extrémité fixe du canal (figure 1). Dans cette région, la gamme du nombre de mobilité est $12<\psi<15$ (nombre de mobilité $\psi=(A \omega)^{2} /[g(s-1) d]$ où $\mathrm{g}$ l'accélération due à la gravité, $\mathrm{d}$ le diamètre de particule, $\mathrm{A}$ l'amplitude orbitale de la houle au fond et $\omega$ la pulsation de la houle). Dans cette zone, la longueur et la hauteur moyenne de ride sont respectivement $l_{\mathrm{r}}=5,5 \mathrm{~cm}$ et $h_{\mathrm{r}}=1,5 \mathrm{~cm}$. Avec ces conditions, le régime d'écoulement est turbulent rugueux.

Dans un premier temps, nous nous sommes intéressés aux trajectoires des particules lors de l'amortissement de l'écoulement. L'expérience menée consiste à saupoudrer des nuages de particules peu denses à travers la surface libre et à capturer la trajectoire de certaines d'entre elles après l'arrêt du batteur. A l'aide d'une mesure de la surface libre par une sonde résistive, le temps d'amortissement de l'écoulement est estimé à $48 \mathrm{~s}$. La fenêtre d'observation contient 4 rides et sa hauteur est d'environ une longueur d'onde de ride $(5,5 \mathrm{~cm})$.

$\mathrm{Au}$ cours d'une deuxième campagne d'essais, nous étudions les trajectoires des particules en dynamique, sous l'action de l'onde stationnaire. On introduit successivement des petites et grosses particules dans la même région du canal et nous étudions leurs trajectoires en particulier à proximité du fond et de la crête de ride.

\section{Modèle théorique}

Le modèle théorique est développé par EZERSKY \& MARIN (2008) dans le but d'expliquer le mouvement de particule près du fond lorsque la houle s'amortit. C'est un modèle physique simple qui considère que le fond sédimentaire est plat. La ligne de courant s'exprime par l'équation: $\psi=-a(\alpha x+y) y$. Les composantes de vitesse $\left(\mathrm{U}_{\mathrm{x}}, \mathrm{U}_{\mathrm{y}}\right)$ de l'écoulement au voisinage de crête de ride sont calculées par les équations suivantes :

$U_{x}=\frac{\partial \psi}{\partial y}=-a(\alpha x+2 y) \quad U_{y}=-\frac{\partial \psi}{\partial x}=a \alpha y$

où $a=a_{0} \sin (\omega t) ; \alpha=\alpha_{0} \sin (\omega t) ;$ a est déterminé par la vorticité $\vec{\Omega}=\operatorname{rot} \vec{U}, a=\Omega / 2$ et $\alpha$ est l'angle de séparation de l'écoulement (EZERSKY \& MARIN, 2008).

On prend en compte seulement la force de Stokes, la turbulence et l'influence visqueuse dans l'équation de mouvement de particule sont négligées. Puisque le nombre de Stokes 
est petit $\left(S t=d_{P V C}^{2} \rho_{P V C} \omega / 18 v \rho_{w} \sim 10^{-3}\right)$. On peut l'utiliser comme paramètre d'expansion. La vitesse de particule peut s'exprimer par :

$$
\begin{aligned}
& \vec{V}=\vec{V}^{(0)}+S t \vec{V}^{(1)}+S t^{2} \vec{V}^{(2)}+\ldots \\
& \vec{V}^{(0)}=\vec{U}-\vec{e}_{y} \frac{d_{P V C}^{2}\left(\rho_{P V C}-\rho_{w}\right) g}{18 v \rho_{w}} \equiv \vec{U}-\vec{e}_{y} U_{0} \\
& \vec{V}^{(1)}=-\frac{1}{\omega}\left(\frac{d\left(\sigma \vec{U}-\vec{e}_{y} U_{0}\right.}{d t}\right) ; \sigma=1-\rho_{w} / \rho_{P V C} \quad U_{0}=\frac{d_{P V C}^{2}\left(\rho_{P V C}-\rho_{w}\right) g}{18 v \rho_{w}}
\end{aligned}
$$

Où : $\vec{V}$ est la vitesse de particule, $v$ la viscosité cinématique du fluide, $\rho$ la densité et les indices $\mathrm{PVC}$ et $\mathrm{w}$ correspondent respectivement à $\mathrm{PVC}$ et fluide; $U_{0}$ est la vitesse sédimentation de Stokes.

On néglige tous les termes proportionnels à $S t^{2}$ dans l'équation (2). Après quelques transformations, on obtient l'expression de deux composantes $V_{x}$ et $V_{y}$ :

$$
\begin{aligned}
& V_{x}=-a(\alpha x+2 y)+\frac{S t}{\omega} \sigma\left[(\dot{a} \alpha+a \dot{\alpha}) x+2 \dot{a} y-a^{2} \alpha^{2} x\right] \\
& V_{y}=a \alpha y-U_{0}-\frac{S t}{\omega} \sigma\left[(\dot{a} \alpha+a \dot{\alpha}) y+a^{2} \alpha^{2} y\right]
\end{aligned}
$$

\section{Résultats}

\subsection{Trajectoires de particules lors de l'amortissement de l'écoulement}

On considère que le temps de référence $t=0$ est le moment où on éteint le batteur. Les notations $t_{1}$ et $t_{2}$ désignent respectivement l'instant de départ où on commence à suivre la trajectoire dans le champ d'observation (début de trajectoire) et l'instant final où la particule se dépose (fin de trajectoire). Le suivi des trajectoires de particules sur un temps long est très délicat. Les trajectoires de particules sont obtenues à l'aide d'un programme développé sous Matlab.

Les périodes d'observation des différentes particules se situent dans la phase d'amortissement de l'onde de surface. Les grosses particules ont une vitesse de chute de l'ordre de 9 fois plus grande que les petites (vitesse de chute $\sim d^{2}$ ). Elles se déposent sur des temps plus courts. Il en résulte des trajectoires assez simples présentant peu d'alternance mais dont l'amplitude de mouvement est grande. Nous avons analysé 63 trajectoires. Bien qu'il soit impossible d'établir un bilan statistique sur ce nombre réduit, il apparaît néanmoins une tendance qui se trouve confirmée par l'observation du tri après arrêt complet du mouvement. L'analyse des trajectoires trouvées montre que $61 \%$ des petites particules (taille : $100-315 \mu \mathrm{m}$ ) tombent au voisinage des crêtes ; 39\% des petites particules tombent ailleurs. Par contre, il y a seulement $29 \%$ des grosses particules (taille : 400-500 $\mu \mathrm{m}$ ) qui tombent au voisinage des crêtes. L'observation d'une tendance des particules à chuter sur la zone de crête est validée pour des 
particules de diamètre $d<315 \mu \mathrm{m}$. En relevant les positions de départ des petites particules dans le champ d'observation, il apparaît que celles-ci sont réparties spatialement de façon relativement homogène. Le tri ne s'effectue qu'au voisinage immédiat de la ride.

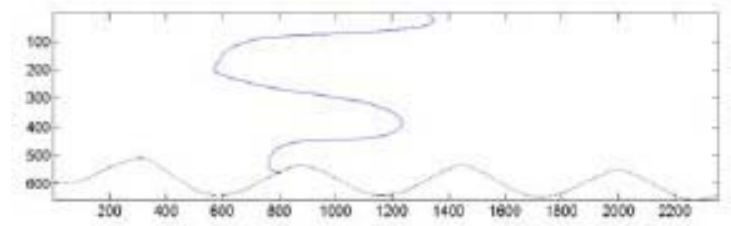

(a)

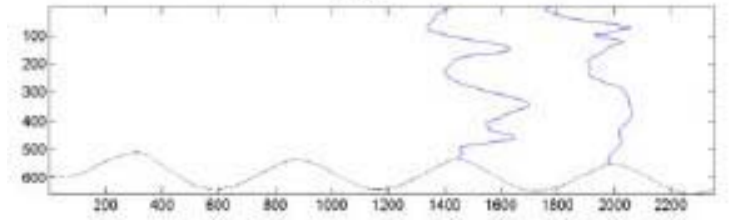

(c)

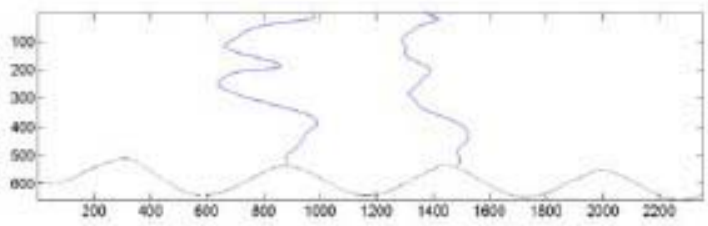

(b)

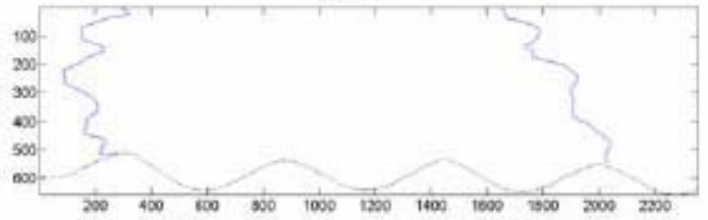

(d)

Figure 2. Trajectoires typiques de particules pour le diamètre :

(a) $d=400-500 \mu \mathrm{m},\left(t_{1}=7,1 \mathrm{~s} ; t_{2}=12,3 \mathrm{~s}\right) ;\left(\right.$ b) $d=250-315 \mu \mathrm{m},\left(t_{1}=14,7 \mathrm{~s} \& 22,2 \mathrm{~s}\right.$; $\left.t_{2}=23,1 \mathrm{~s} \& 31,1 \mathrm{~s}\right) ;(c) d=125-250 \mu \mathrm{m},\left(t_{1}=14,8 \mathrm{~s} \& 21,4 \mathrm{~s} ; t_{2}=26,2 \mathrm{~s} \& 32,5 \mathrm{~s}\right)$; (d) $d=100-125 \mu m,\left(t_{1}=22,1 s \& 25,8 s ; t_{2}=35,5 s \& 35,4 s\right)$

(Coordonnées en pixels; 100 pixels $\sim 1 \mathrm{~cm}$ ).

\subsection{Comparaison des trajectoires théorique et expérimentale}

Après l'arrêt du batteur, l'écoulement s'amortit avec le temps. On considère que les deux paramètres $a$ et $\alpha$ diminuent de la façon suivante : $a=a_{0} \sin (\omega t) \times e^{-y t} ; \alpha=\alpha_{0} \sin (\omega t) \times e^{-\gamma t}$, où $\gamma$ est le taux exponentiel d'amortissement de la surface libre. Le temps d'amortissement de la surface libre $T_{d} \approx 48 \mathrm{~s}$, le taux exponentiel de dégradation de la surface libre $\gamma=1 / T_{d}=0,021\left[\mathrm{~s}^{-1}\right]$. La pulsation de la houle est $\omega=1,96[\mathrm{Rad} / \mathrm{s}]$ et $\sigma=0,26$; On estime $a_{0}=0,3\left[\mathrm{~s}^{-1}\right]$ et $\alpha_{0} \approx 1$ [rad]. Pour une particule PVC de $d=250 \mu \mathrm{m}, U_{0}=0,012[\mathrm{~m} / \mathrm{s}]$ et $S t=0,0092$.
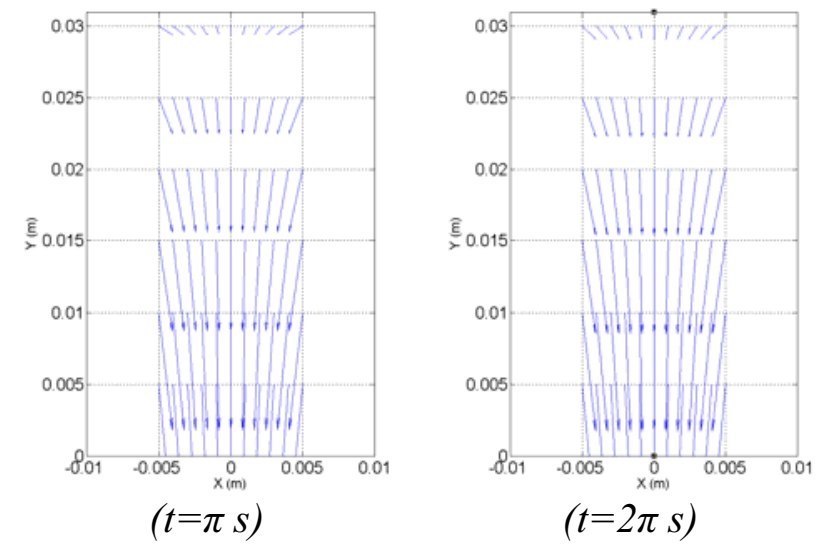

Figure 3. Champ de vitesse d'une particule $d=250 \mu \mathrm{m}$. 
Sur la figure 3, on trouve que pour la partie amont (à gauche) de la crête $(X<0)$, la composante horizontale $V_{x}>0$ et pour la partie avale de la crête $(X>0), V_{x}<0$. Les particules tendent donc à se concentrer à la crête de ride $(X=0, Y=0$ : point hyperbolique).

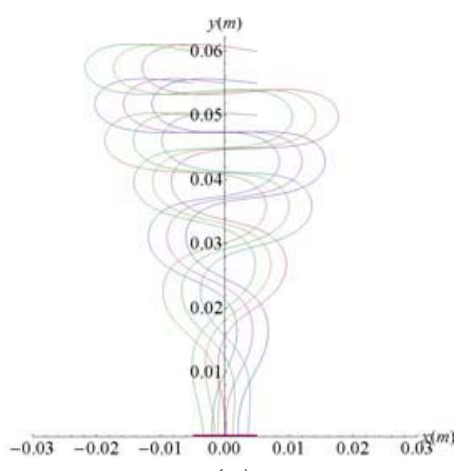

(a)

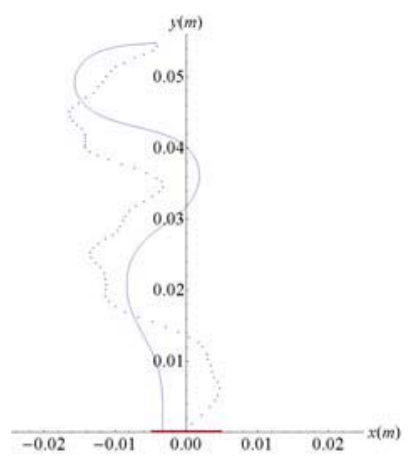

(b)

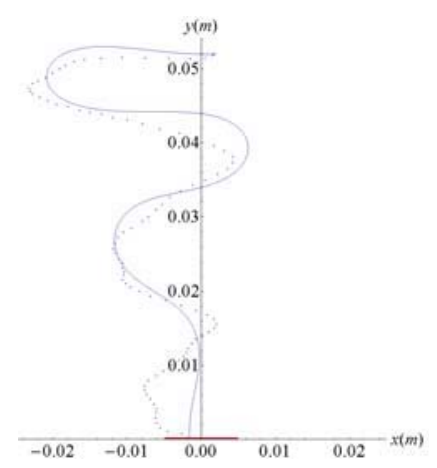

(c)

Figure 4. (a) Trajectoires théoriques $(d=250 \mu \mathrm{m})$ avec 9 points de départ

(b) (c) Comparaison des trajectoires théorique et expérimentale $(d=250 \mu \mathrm{m})$

Dans la figure 4, la ligne rouge au fond représente le voisinage de la crête $(1 \mathrm{~cm}$ de longueur). La figure 4(a) montre que les petites particules se concentrent à la crête de ride. On a un bon accord qualitatif entre les trajectoires issues du modèle théorique et les trajectoires expérimentales (figures 4(b) et 4(c)) pour les particules dont le diamètre est inférieur à $315 \mu \mathrm{m}$.

\subsection{Zone de confinement - Amélioration du modèle théorique}

Sous l'action d'un écoulement oscillant, les tourbillons sur les flancs des rides sont générés à chaque alternance de l'écoulement. Ces structures organisées sont des agents hydrodynamiques dominants près du fond. En dynamique, des particules situées aux creux et aux flancs sont transportées par les tourbillons vers les crêtes (figure 5(a)). Très rapidement, on peut observer le mouvement alternatif de grains dans une zone qui reste confinée au voisinage des crêtes.

Dans la zone de confinement, les particules bougent périodiquement avec la même pulsation que celle de la houle. Les particules peuvent être capturées et éjectées par cette zone de confinement (figures 5(b) et 5(c)). Les tests effectués montrent que les particules éjectées sont les grosses particules, dont la quantité de mouvement est plus importante et qui sont plus exposées aux tourbillons que les autres. La figure 5(d) représente la zone de confinement construite à partir des positions extrêmes des particules présentes dans la zone. Nous pouvons estimer la hauteur et la largeur de la zone de confinement : $\mathrm{h}_{\mathrm{zc}}=0,85 \mathrm{~mm}$ et $L_{\mathrm{zc}}=10,92 \mathrm{~mm}$. Cette hauteur est comparable à l'épaisseur de la couche de Stokes $\delta=0,5 \mathrm{~mm}$ et la largeur $L_{\mathrm{zc}} \sim l_{\mathrm{r}} / 5$, où $1_{\mathrm{r}}$ est la longueur moyenne de ride dans la zone testée. 


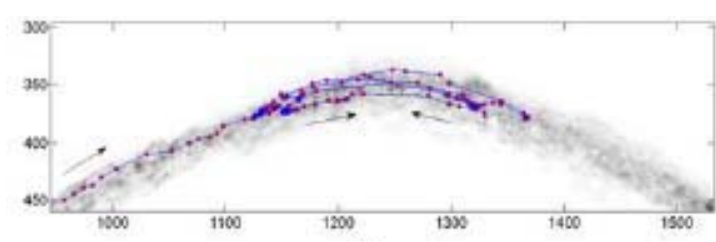

(a)

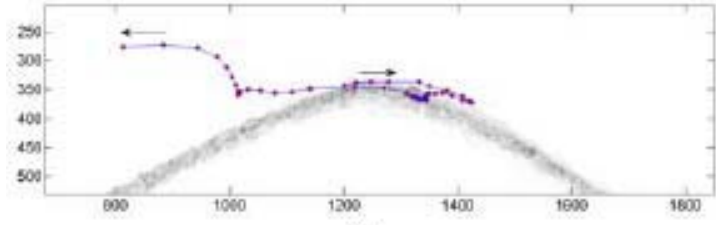

(c)

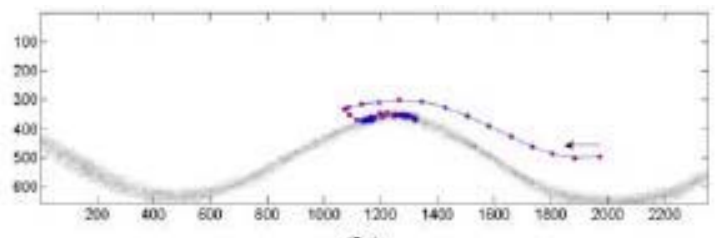

(b)

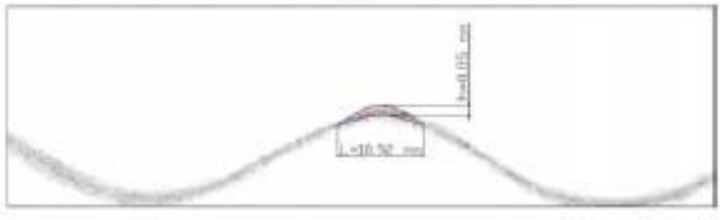

(d)

Figure 5. Mise en évidence d'une zone de confinement au voisinage de la crête

(a) Mouvement alternatif d'une particule au sommet ( $d=245-262 \mu \mathrm{m})$

(b) Capture d'une particule en suspension ( $d=234-251 \mu \mathrm{m})$

(c) Ejection d'une particule ( $d=290-304 \mu \mathrm{m})$

(d) Dimensions de la zone de confinement $\left(L_{z c}=10,92 \mathrm{~mm} ; h_{z c}=0,85 \mathrm{~mm}\right)$.

A partir de l'observation de cette zone, nous trouvons qu'il existe un mouvement alternatif des petites particules aux sommets des rides en phase dynamique. Cela suggère que le point hyperbolique $(X=0, Y=0)$ dans la partie théorique précédente bouge alternativement en fonction du temps. En réalité, ce point bouge sur la courbure de crête de ride, mais dans le modèle théorique, on considère que le point bouge alternativement sur une ligne horizontale. Dans ce cas, l'expression du potentiel et les composantes de la vitesse d'écoulement deviennent :

$\psi=-a\left[\alpha\left(x+x_{0}\right) y+y^{2}\right] ; U_{x}=\frac{\partial \psi}{\partial y}=-a\left[\alpha\left(x+x_{0}\right)+2 y\right] ; U_{y}=-\frac{\partial \psi}{\partial x}=a \alpha y$

où : $x_{0}=X_{0} \times \sin (\omega t)$ représente le point de crête oscillant avec le temps. Les composantes de la vitesse de particule peuvent alors s'écrire :

$V_{x}=-a\left[\alpha\left(x+x_{0}\right)+2 y\right]+\frac{S t}{\omega} \sigma\left[(\dot{a} \alpha+a \dot{\alpha})\left(x+x_{0}\right)+2 \dot{a} y-a^{2} \alpha^{2}\left(x+x_{0}\right)+a \alpha \dot{x}_{0}\right]$

$V_{y}=a \alpha y-U_{0}-\frac{S t}{\omega} \sigma\left[(\dot{a} \alpha+a \dot{\alpha}) y+a^{2} \alpha^{2} y\right]$

En utilisant les équations (6) et (7), on modélise quelques trajectoires théoriques avec un point d'oscillation $x_{0}$ et on compare les trajectoires théoriques du modèle initial et du modèle modifié lors de l'amortissement de l'écoulement.

La figure 6 montre que le modèle théorique avec le point d'oscillation modélise mieux le phénomène de dépôt à la crête des petites particules qu'avant. Des particules qui ne sont pas initialement situées au dessus de la crête sont ramenées à la crête. Le point d'oscillation apporte une bonne adaptation à notre cas. 


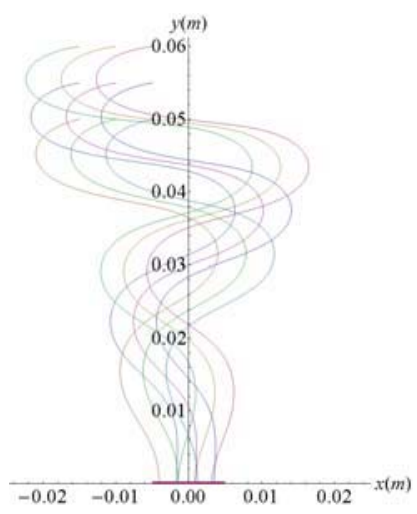

(a)

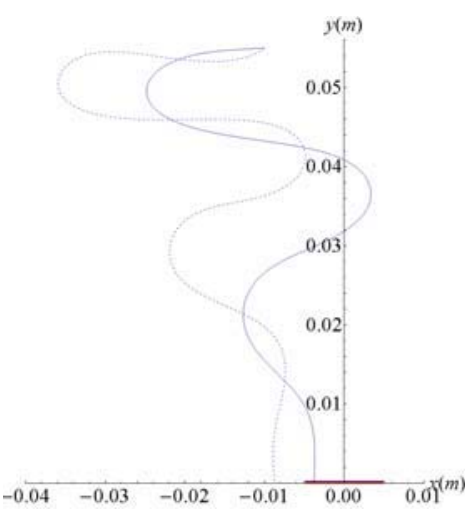

(b)

Figure 6. Modélisation des trajectoires théoriques $(d=250 \mu \mathrm{m})$ avec le point d'oscillation $x_{0}=X_{0} \times \sin (\omega t) e^{-\gamma t}$ où $X_{0}=0,005 \mathrm{~m}$. (a) : 9 points de départ,

(b) : Trajectoires avec oscillation du point hyperbolique - courbe continue et avec point hyperbolique fixe - courbe en trait tireté (EZERSKY \& MARIN, 2008).

\section{Conclusions}

Dans cette expérience, nous nous intéressons aux trajectoires de particules en suspension sous l'action d'un écoulement oscillant au dessus d'un fond ridé. Lorsque l'écoulement s'amortit, on observe que les petites particules tendent à se concentrer au voisinage des crêtes de ride alors que les grosses particules se déposent indifféremment le long de la ride. Le modèle théorique résultant d'une amélioration du modèle initialement proposé par EZERSKY \& MARIN (2008) modélise de façon satisfaisante les trajectoires et la zone de dépôt des petites particules. Nous obtenons un bon accord entre les résultats issus du modèle théorique et les résultats expérimentaux.

Remerciements: Le travail est effectué dans le cadre d'une thèse financée par la Région Haute-Normandie (Réseau REIVE).

\section{Références bibliographiques}

BALASUBRAMANIAN S., VOROPAYEV S.I., FERNANDO H.J.S. (2008). Grain sorting and decay of sand ripples under oscillatory flow and turbulence. Journal of Turbulence, Vol.9, No. 17, pp 1-19.

EZERSKY A.B., MARIN F. (2008). Segregation of sedimenting grains of different densities in an oscillating velocity field of strongly nonlinear surface waves. Physical Review E, Vol.78, No 2, 4 p. doi:10.1103/PhysRevE.78.022301

FARACI C., FOTI E. (2002). Geometry, migration and evolution of small scale bedforms generated by regular and irregular waves. Coastal Engineering 47, pp 35-52. doi:10.1016/S0378-3839(02)00097-2

NIELSEN P. (1981). Dynamics and geometry of wave generated ripples. Journal of Geophysical Research 86(7), pp 6467-6472. doi:10.1029/JC086iC07p06467 